\title{
Carcinoma de células escamosas bucal: uma revisão de literatura entre o perfil do paciente, estadiamento clínico e tratamento proposto
}

\author{
Oral squamous cell carcinoma: a literature review of patient profile, clinical \\ staging and proposed treatment
}

Sylvie Brener', Franca Arenare Jeunon², Alvimar Afonso Barbosa ${ }^{3}$, Helenice de Andrade Marigo Grandinetti ${ }^{4}$

\section{Resumo}

O carcinoma de células escamosas (CEE) é uma neoplasia maligna, com origem no epitélio de revestimento da boca, sendo responsável por cerca de $95 \%$ das lesōes malignas nesta região. No Brasil, a boca representa a quinta localização de maior incidência de câncer em homens e a sétima em mulheres. Este trabalho teve como objetivo analisar as variáveis: perfil (gênero, cor, idade, ocupação e escolaridade), fatores etiológicos (tabagismo, etilismo e exposição ao sol), com o estadiamento clínico (sistema TNM) e, conseqüentemente, o tratamento indicado. Foram incluídos 75 artigos. Verificou-se um predomínio das lesões na língua (32,7\%), seguido pelo assoalho bucal (23,3\%). A lesão foi predominantemente encontrada em feodermas, entre a $5^{\mathrm{a}}$ e a $7^{\mathrm{a}}$ décadas de vida. No momento do diagnóstico, a maioria dos pacientes se encontrava em estádio avançado da lesão (III ou IV). Os fatores mais associados ao desenvolvimento do CCE bucal foram: tabagismo, etilismo e radiação solar, considerando-se que estes podem complicar seu curso e prognóstico. Verificou-se que quanto maior o nível do estadiamento clínico, mais complexo foi o tratamento indicado.

Palavras-chave: Carcinoma de células escamosas bucal; Epidemiologia; Perfil do paciente; Estadiamento clínico; Tratamento.

\footnotetext{
${ }^{1}$ Mestre em Estomatologia; Professora da Faculdade de Odontologia da PUC-MG; Doutoranda em Patologia na FOB-USP.

${ }^{2}$ Mestre em Estomatologia; Coordenadora do Curso de Odontologia da PUC-MG.

${ }^{3}$ Professor da Faculdade de Medicina da UFMG.

${ }^{4}$ Doutora em Patologia; Professora da Faculdade de Odontologia da PUC-MG.

Endereço para correspondência: Sylvie Brener. Disciplina de Estomatologia - Laboratório de Biologia Oral - Faculdade de Odontologia - PUC-MG. Av. Dom José Gaspar 500 - Bairro Coração Eucarístico - Belo Horizonte - MG - Brasil - CEP: 30535-610. E-mail: sylviebrener@globo.com
} 


\section{INTRODUÇÃO}

O carcinoma de células escamosas (CCE) da boca, também denominado carcinoma epidermóide, carcinoma escamocelular e carcinoma espinocelular, é uma neoplasia maligna que se origina no epitélio de revestimento, sendo considerada a neoplasia maligna mais comum nesta região.

As taxas de incidência e mortalidade para o CCE bucal variam de um país para outro e mesmo dentro de cada país. Essas variações ocorrem, principalmente, pelas diferenças de hábitos, características socioeconômicas, expectativa de vida, fatores ambientais, raça, educação preventiva e qualidade da assistência médica nas diversas regiōes.

A distribuição das taxas de incidência e de mortalidade de tumores malignos, inclusive o CCE bucal, tem sido, em muitos países, de grande importância para o estabelecimento de diretrizes de políticas públicas em âmbito nacional, estadual e municipal e para o planejamento de ações de prevenção e controle do câncer, além de formas de tratamento ou assistência terapêutica ${ }^{1}$. Dados estatísticos são essenciais aos programas de vigilância em saúde pública, pois permitem estimar a magnitude relativa dos problemas de saúde na população, facilitando o estabelecimento de prioridades em ações preventivas e terapêuticas.

A abordagem do CCE bucal torna-se complexa, pois, muitas vezes, enfrenta-se o desconhecimento e a falta de recursos dos profissionais de saúde, além de envolver o medo e o preconceito dos pacientes. Essas intercorrências são prejudiciais, atrasando o diagnóstico e o tratamento, e piorando o prognóstico.

\section{DISTRIBUIÇÃO E INCIDÊNCIA}

Anualmente, são diagnosticados cerca de 6,4 milhões de casos de tumores malignos no mundo, sendo o câncer bucal responsável por $10 \%$ dos casos ${ }^{2}$. No Brasil e no Reino Unido, o CCE responde por $90 \%$ a $95 \%$ dos tumores malignos da boca ${ }^{2,3}$.

A estimativa para o ano de 2006, em todo o Brasil, seria de ocorrerem 472.570 novos casos de câncer, sendo que, desses, 13.470 seriam câncer de boca ${ }^{2}$. Para o Estado de Minas Gerais, eram esperados 1.030 novos casos de CCE bucal, sendo 750 destes em homens e 280 em mulheres. Para Belo Horizonte, a previsão era de 200 novos casos ${ }^{2}$.

Estudo realizado, comparando o Brasil e a Índia, citando dados da Agência Internacional de Pesquisa do Câncer (IARC), afirmou que o país asiático possui a maior taxa de incidência de câncer bucal do mundo, seguido pelo Brasil ${ }^{4}$.

\section{Perfil do PaCiente Portador de carcinoma bucal de CÉLULAS ESCAMOSAS (CCE)}

Conhecer o perfil dos portadores de CCE bucal é muito importante para auxiliar o direcionamento das campanhas de prevenção, especialmente em populações com características socioeconômicas e culturais tão específicas quanto a que é atendida na instituição-alvo deste estudo.

\section{Diferença entre gêneros}

Esperava-se, no Brasil, para o gênero masculino, 10.060 novos casos em 2006, enquanto que a estimativa para o gênero feminino era de 3.410 novos casos para o mesmo ano, representando o sétimo tipo de câncer mais freqüente em homens e o nono em mulheres ${ }^{2}$.

Em um levantamento realizado no prontuário de 740 pacientes nos Hospitais Mário Penna e Luxemburgo, em Belo Horizonte (MG), observou-se que $82,7 \%$ dos pacientes com CCE bucal eram do sexo masculino e $17,3 \%$ do sexo feminino, sendo que a razão homem:mulher foi de $4,8: 1^{5}$.

Com a mudança no comportamento feminino, que passou a se expor mais a associação álcool-tabaco, houve um aumento do CCE bucal nas mulheres, com conseqüente redução da razão homem:mulher ${ }^{6,7}$. Tal afirmativa é confirmada pela estimativa mais recente do INCA que prevê 2,95 casos em homens para cada mulher diagnosticada ${ }^{2,7}$.

\section{Cor e raça}

Uma pesquisa realizada em São Paulo, Curitiba e Goiânia encontrou 83,2\% dos casos de CCE bucal em brancos, $11,2 \%$ em mulatos e $4,3 \%$ em negros $^{8}$.

Entre os pacientes examinados em Belo Horizonte, $33,5 \%$ eram negros, $23,7 \%$ brancos e $42,8 \%$ mulatos $^{5}$.

Em estudo realizado nos Estados Unidos, verificouse que $80,6 \%$ dos pacientes com CCE bucal eram leucodermas, $18,1 \%$ afro-americanos e $1,3 \%$ asiáticos ou hispânicos? .

Uma pesquisa realizada em Belo Horizonte observou que os pacientes com CCE bucal tinham entre 40-80 anos de idade, sendo que $32,5 \%$ encontravam-se na $6^{a}$ década de vida 5 . A média de idade obtida, no momento do diagnóstico, foi de 58,6 anos (58,2 anos para homens e 63,9 anos para mulheres) 5 .

O National Cancer Institute (NCI, 2004) relatou que $90 \%$ dos portadores de CCE bucal tinham mais de 45 anos $^{10}$. 


\section{Procedência e ocupação}

Em um estudo realizado no Brasil, cerca de 76,3\% dos portadores de CCE bucal procediam de área rural e $23,7 \%$ de área urbana ${ }^{8}$.

Encontrou-se o CCE de lábio acometendo mais os homens do que as mulheres e afetando, principalmente, a população rural ${ }^{3}$.

Estudo realizado em Belo Horizonte verificou que $80,5 \%$ dos pacientes incluídos procediam de áreas urbanas $^{11}$.

Autores sugerem que pessoas procedentes de área rural teriam acesso mais difícil ao tabaco e à bebida alcoólica, por isto o menor número de consumidores entre as pessoas com esta origem?.

Pesquisa realizada em São Luís (Maranhão) encontrou $56,6 \%$ dos portadores de CCE bucal procedentes do interior, $34,5 \%$ da capital e $4,1 \%$ de outros estados. Essa mesma pesquisa revelou que, entre os portadores de CCE bucal, 31\% eram lavradores e $18,7 \%$ eram domésticas ${ }^{12}$.

A ocupação mais comumente encontrada em portadores de CCE bucal foi a de trabalhador rural (36,2\% dos pacientes $)^{5}$.

Nenhuma profissão pôde ser diretamente relacionada ao risco de desenvolver o CCE bucal ${ }^{7}$. Mulheres apresentavam uma menor diversidade de ocupações do que os homens, estando mais ligadas ao trabalho do lar e à lavoura ${ }^{6}$.

\section{Fatores socioeconômicos e culturais \\ Escolaridade}

Os analfabetos constituíram $44,8 \%$ dos portadores de CCE bucal incluídos em uma amostra ${ }^{11}, 48,8 \%$ de outra $^{13}$, alcançando $74 \%$ em outros estudos ${ }^{8,14}$.

\section{Renda}

Estudo, revisando publicações sobre o câncer bucal no Brasil, encontrou relação positiva entre esta neoplasia e baixo nível socioeconômico ${ }^{15}$. Esta relação não pôde ser confirmada quando se envolveu a escolaridade e o estadiamento clínico realizado no momento do exame inicial ${ }^{15}$.

\section{Fatores de risco}

\section{Tabaco}

Cerca de 90\% dos indivíduos com diagnóstico de CCE bucal consumiam tabaco sob a forma de cigarro, charuto, cachimbo ou mascado e, entre os que relatavam nunca ter fumado, a proporção homem:mulher se invertia, com as mulheres representando a maioria do grupo ${ }^{13,16}$.

O risco de desenvolvimento de CCE bucal em fumantes de cigarro industrializado é 6,3 vezes maior do que em não-fumantes. Este risco aumentaria para sete vezes em consumidores de cigarro de palha, e para 14 vezes em usuários de cachimbo ${ }^{8}$. No entanto, cerca de 4,3\% dos portadores de CCE bucal nunca haviam fumado ${ }^{8,13}$.

A redução do risco de desenvolvimento do câncer bucal em um nível próximo ao dos que nunca fumaram é percebida dez anos após a interrupção do hábito de fumar ${ }^{8,17,18}$. Essa redução do risco pode ser de 15 anos para ex-fumantes de cigarro industrializado e 20 anos para ex-fumantes de cigarro feito com fumo de rolo ${ }^{19}$.

O consumo de cigarro vem se reduzindo desde o início da década de 1970, apesar de ainda ser encontrado em níveis alarmantes ${ }^{20}$. Dados divulgados sobre o Brasil revelaram que $31 \%$ dos adultos (considerados aqueles acima de 15 anos de idade) utilizavam cigarro, atingindo o consumo per capita de 858 unidades, no ano 2000, devendo-se considerar que este valor não incluiu os cigarros adquiridos por contrabando ${ }^{20}$.

\section{Álcool}

O consumo de $0,5 \mathrm{~L}$ a 1 litro de vinho por dia proporcionaria um risco 34 vezes maior para o desenvolvimento do CCE bucal ${ }^{21}$.

Entre os portadores de CCE bucal que eram usuários de bebidas alcoólicas, $70 \%$ consumiam bebidas destiladas, mais especificamente a cachaça, sugerindo uma participação diferenciada desse tipo de bebida na carcinogênese bucal ${ }^{13,14}$.

Enquanto $29 \%$ das mulheres relataram-se etilistas, este número foi de $92 \%$ entre indivíduos do gênero masculino ${ }^{22}$.

\section{Tabaco e álcool}

No Brasil, o CCE bucal é mais associado ao tabaco sob a forma de cigarro para fumar e à bebida alcoólica, enquanto na Índia haveria uma forte relação com o fumo mascadó .

Para enfatizar a importância da participação do tabaco e do álcool no desenvolvimento do câncer bucal, um estudo verificou estes hábitos e encontrou 92,2\% de fumantes e $81,2 \%$ de etilistas 9 . O efeito simultâneo do álcool e do tabaco pode aumentar em até 100 vezes o risco de se desenvolver um câncer bucal ${ }^{23}$.

O álcool sozinho não pode ser associado à fase iniciadora da carcinogênese bucal, mas acredita-se que ele possa promovê-la ao potencializar os efeitos carcinógenos do tabaco ${ }^{18}$.

O etilismo isolado ou associado ao tabagismo afeta a incidência de CCE bucal, proporcionando aumento 
na incidência em faixas etárias mais precoces, tanto nas mulheres quanto nos homens ${ }^{22}$.

\section{Luz solar}

A exposição contínua ao sol está associada ao câncer do vermelhão labial, que se desenvolve a partir de uma lesão cancerizável, denominada queilite actínica ${ }^{23}$. O CCE labial inicia-se como um processo crônico e a exposição contínua à luz solar favorece o desenvolvimento da queilite actínica que se transforma em carcinoma de células escamosas ${ }^{24}$.

\section{Localização do tumor}

A língua é o sítio mais comum para o CCE bucal, correspondendo a $44 \%$ dos casos, seguido pelo assoalho bucal com 16\% ${ }^{5}$. Verificou-se maior prevalência desta lesão no assoalho bucal (27,9\%), seguido pela língua $(22,1 \%)$ e pelo trígono retromolar $(15,6 \%)^{11}$.

A localização anatômica foi considerada fator de influência no prognóstico, considerando-se que os tumores apresentam comportamento clínico diferente, conforme a sua localização ${ }^{25}$.

A mucosa jugal foi o sítio de maior incidência em pacientes acima de 60 anos. Em pacientes mais jovens, houve maior acometimento da língua, isolada ou associada ao assoalho bucal ${ }^{22}$.

\section{Estadiamento clínico}

Entre os anos de 1943 e 1952, o francês Pierre Denoix desenvolveu o sistema TNM para a classificação de tumores malignos, tendo sido incorporado pelo AJCC e pela UICC a partir de 1982, com a finalidade de unificar a linguagem dos oncologistas ${ }^{26}$. A classificação por esse sistema dependeria de três características: tamanho do tumor (T), em centímetros; acometimento dos linfonodos e sua extensão $(\mathrm{N})$; e presença ou não de metástases distantes $(\mathrm{M})$. Quanto maior a classificação do estadiamento, pior o prognóstico ${ }^{26}$.

O sistema de estadiamento clínico TNM permite avaliar as características fundamentais do CCE bucal: extensão local, disseminação regional e metástase à distância ${ }^{25}$. Neste estudo, 55\% dos pacientes tiveram suas lesões diagnosticadas em fase avançada, porém aqueles com lesão de lábio inferior apresentavam-se em fase inicial (I ou II), ressaltando que a localização influencia o curso da doençâa ${ }^{25}$.

\section{Tratamento}

O impacto do atendimento por médicos especialistas para vários tipos de câncer, entre eles o de boca, sugere que estes pacientes podem ter um aumento médio na sobrevida global em torno de $5 \%$ a $10 \%$. Para estes autores, a modalidade terapêutica mais indicada para o CCE bucal seria a cirurgia ${ }^{28}$.

Ao preconizar o tratamento radioterápico associado a uma dose única e alta de quimioterapia (QT), utilizando a cisplatina como agente único, propõe-se elevar para $37 \%$ a sobrevida em três anos quando comparado aos $23 \%$ para aqueles submetidos à radioterapia (RT) exclusiva $^{29}$.

Alguns médicos preconizam a radioterapia concomitante à quimioterapia no pré-operatório, pela redução do volume tumoral, apesar de os efeitos colaterais serem substanciais e nem sempre aceitáveis para os pacientes ${ }^{30}$.

\section{Cirurgia}

A cirurgia deve permanecer como terapia de escolha para o CCE bucal, sendo que a modalidade varia de acordo com a extensão clínica ou estádio da doença na apresentação, variando de uma excisão local até uma remoção mais ampla ${ }^{24,31}$.

Relata-se a ocorrência de metástase cervical oculta em $10 \%$ a $26 \%$ dos pacientes, justificando a indicação do tratamento efetivo do pescoço ${ }^{32}$.

Relata-se que $40 \%$ a $45 \%$ dos pacientes com lesão em fases III ou IV, consideradas passíveis de remoção pela cirurgia, apresentariam recidiva loco-regional ou à distância ${ }^{24,33}$.

\section{Radioterapia (RT)}

A RT deve ser a modalidade terapêutica de escolha para o CCE bucal, quando o paciente não apresentar condiçôes clínicas para ser submetido à cirurgia ou não aceitar os possíveis defeitos que esta pode deixar ${ }^{33}$.

Todos os pacientes com CCE bucal avançado necessitam de RT adjuvante, sendo ela pré ou pósoperatória, sugerindo que a RT pré-operatória aumenta o risco de complicaçôes na cirurgia ${ }^{31}$.

A RT pré-operatória não traz vantagens para a sobrevida do paciente, além de dificultar a realização da cirurgia em um intervalo menor do que seis semanas após o término da $\mathrm{RT}^{3}$.

\section{Quimioterapia (QT)}

A QT adjuvante ou paliativa para lesōes primárias de CCE bucal, quando estas se apresentam muito grandes ou irressecáveis, tem sido indicada sem que se tenha verificado redução na taxa de mortalidade ou melhora no prognóstico ${ }^{24}$.

Sugeriu-se um protocolo baseado na administração de cisplatina intra-arterial concomitantemente à radiação 
em pacientes com CCE bucal. Foram encontradas respostas completas em $75 \%$ dos pacientes, resposta parcial em $23 \%$ e ausência de resposta em $2 \%$ dos pacientes ${ }^{32}$.

Considerando-se que a QT apresenta resultados imprevisíveis, no Reino Unido ela é indicada somente para CCE bucal no estádio IV, inoperável ou recorrente, associada ou não à $\mathrm{RT}^{3}$.

\section{Cirurgia vs. RT vs. QT}

A cirurgia permanece como primeira opção de tratamento do CCE bucal, devendo a RT ser indicada quando a lesão não é removida cirurgicamente com margem de segurança ou quando o tumor se apresenta em fase avançada no momento do diagnóstico. Entre $40 \%$ e $45 \%$ dos pacientes com lesão em fase III ou IV, consideradas passíveis de remoção pela cirurgia, apresentam recidiva loco-regional ou à distância. Para este grupo indica-se QT, porém, avaliando os resultados, não houve benefício significativo em termos de aumento na sobrevida ${ }^{24,33}$.

Dedivitis et al. (2004) realizaram levantamento na cidade de Santos (São Paulo), no qual constataram que, entre os pacientes diagnosticados com CCE bucal, 20 (47\%) haviam sido submetidos à RT adjuvante pósoperatória e os demais 23 casos (53\%) à cirurgia exclusiva. Não foi constatada diferença entre a modalidade de tratamento e a sobrevida.

A análise de 957 (29,3\%) pacientes submetidos à cirurgia exclusiva, 1551 (47,5\%) à RT exclusiva, e 759 $(23,7 \%)$ à terapia combinada (cirurgia seguida de RT) permitiu observar um aumento na sobrevida global de cinco anos, comparando os pacientes tratados na década de $1950(28,7 \%)$ com os atendidos nos anos 1990 $(43,2 \%)$. Este fato foi associado a uma mudança na abordagem terapêutica, que envolveu aumento do número de casos submetidos à cirurgia (associada ou não à RT) ${ }^{7}$.

\section{Tratamento vs. estadiamento clínico}

Sugerem-se três modalidades básicas de tratamento para o CCE bucal: cirurgia, RT (feixes externos ou implantes) e quimioterapia. A escolha da modalidade depende do estádio, do tipo do tumor, do envolvimento ósseo, da saúde geral do paciente e da capacitação do especialista ${ }^{3,34}$.

Estádio I: cirurgia ou RT produziriam resultados semelhantes;

Estádio II: cirurgia ou RT, provavelmente, com resultados igualmente positivos, mas a maioria deveria ser submetida à cirurgia inicialmente;

Estádio III: cirurgia, depois RT;

Estádio IV: cirurgia, depois RT.

\section{CONCLUSÕES}

As taxas de incidência e de mortalidade em decorrência do CCE bucal apresentaram variaçōes entre as diferentes regiōes do país.

Constatou-se uma diminuição da taxa de incidência homem:mulher, devido ao aumento na incidência do CCE bucal em mulheres. Admite-se que esta alteração seja devido à mudança no comportamento feminino, que passou a ingerir mais a associação álcool-tabaco.

$\mathrm{O}$ acometimento de determinada cor/raça variou conforme a metodologia aplicada no estudo e a região onde o mesmo foi realizado.

Pessoas acima de 40 anos de idade constituíram grupo predominante em todos os estudos, porém observou-se maior acometimento de homens em idade mais precoce do que de mulheres, sendo que estas predominaram no grupo acima de 70 anos.

Uma mudança no perfil dos portadores de CCE bucal pôde ser observada em relação à procedência dos pacientes, pois estudos mais recentes detectaram maioria com relato de origem urbana. Nenhuma ocupação foi diretamente associada ao desenvolvimento do câncer bucal.

Todos os estudos encontraram baixa escolaridade entre os participantes.

A participação do tabaco na carcinogênese bucal é uma unanimidade entre os autores, mas esta variou em função do tipo de tabaco (fumado, mascado, em pó, adicionado ou não a substâncias adoçantes e aromatizantes), do tempo relatado do hábito, idade de início e associação ou não com o etilismo. Segundo alguns autores, o álcool não agiria sozinho na fase iniciadora da carcinogênese bucal, mas atuaria potencializando os efeitos do tabaco.

Os raios UV do sol foram associados ao desenvolvimento do CCE labial, principalmente entre aqueles pacientes que apresentavam pele clara e exposição ocupacional ao sol.

As localizaçôes mais comumente relacionadas ao CCE bucal foram a língua, o assoalho bucal e o lábio inferior.

$\mathrm{O}$ estadiamento clínico influencia a escolha do tratamento e o prognóstico dos pacientes, sendo que a maioria se apresentava em fase avançada da doença no momento do diagnóstico.

\section{REFERÊNCIAS}

1. Araújo Filho VJF, De Carlucci JD, Sasaki SU. Perfil da incidência do câncer oral em um hospital geral em São Paulo. Rev Hosp Clin Fac Med São Paulo. 1998;55(3):110-13.

2. Instituto Nacional de Câncer (INCA/MS) [homepage na 
internet]. Estimativa da incidência e mortalidade por câncer no Brasil. [acesso em set 2005]. Disponível em: <http:// www.inca.gov.br>

3. Worrall SF. Oral cancer [cited 2003 Apr 26]. Available from: $<$ http://millennium3.org.uk>

4. Hamada GS, Bos AJ, Kasuga H, Hirayama T. Comparative epidemiology of oral cancer in Brazil and in India. Tokai J Exp Clin Med. 1991;16(1):63-72.

5. Gervásio OLAS, Dutra RA, Tartaglia SMA, Vasconcelos WA, Barbosa AA, Aguiar MCF. Oral squamous cell carcinoma: A retrospective study of 740 cases in a Brazilian Population. Braz Dent J. 2001;12(1):57-61.

6. Amorim Filho FS. Estudo de variáveis demográficas, ocupacionais e co-carcinogenéticas no carcinoma espinocelular da base de língua nas mulheres. Rev Bras Otorrinolaringol. 2003;69(4):472-78.

7. Carvalho AL, Ikeda MK, Magrin J. Trends of oral and oropharyngeal cancer survival over five decades in 3267 patients treated in single institution. Oral Oncol. 2004;40:71-76.

8. Franco EL, Kowalski LP, Oliveira BV, Curado MP, Pereira RN, Silva ME, et al. Risk factors for oral cancer in Brazil: a case-control study. Int J Cancer. 1989;43(6):992-1000.

9. Miller CS, Henry RG, Rayens MK. Disparities in risk of and survival from oropharyngeal squamous cell carcinoma. Oral Surg Oral Med Oral Pathol Oral Radiol Endod. 2003;95(5):570-75.

10. National Cancer Institute. Lip and oral cavity cancer. [cited 2004 Ago 5]. Available from: <http://www.meb.unibonn.de/cancer.gov>

11. Abdo EN. Perfil do paciente portador de carcinoma epidermóide da cavidade bucal em tratamento num hospital vinculado ao Sistema Único de Saúde em Belo Horizonte [Dissertação de Mestrado]. Belo Horizonte (MG): Universidade Federal de Minas Gerais; 2001.

12. Silva B, Cutrim MCFN, Nascimento MDSB. Aspectos epidemiológicos do carcinoma epidermóide da cavidade oral: casuística do Instituto Maranhense de Oncologia Aldenora Bello em São Luís (Maranhão). Rev Hosp Universitário/UFMA. 2002;1(4):11-15.

13. Reis SRA. Fatores de risco do câncer de cavidade oral e da orofaringe: fumo, álcool e outros determinantes. RPG Rev Pos-grad. 1997;4(2):127-32.

14. Leite ICG, Koifman S. Survival analysis in a sample of oral cancer patients at a reference hospital in Rio de Janeiro, Brazil. Oral Oncol. 1998;34(5):347-52.

15. Wünsch Filho V. The epidemiology of oral and pharynx cancer in Brazil. Oral Oncol. 2002;38:737-46.

16. Clarke L. Increasing public awareness of oral cancer profile. Baltimore: Spring; 2002.

17. Blot WJ, McLaughlin JK, Winn DM, Austin DF, Greenberg RS, Preston-Martin S, et al. Smoking and drinking in relation to oral and pharyngeal cancer. Cancer
Res. 1988;48(11):3282-287.

18. Macpherson LMD, Gibson J, Binnie VI, Conway DI. Oral cancer: aetiology. Glasgow: University of Glasgow; 2003 [cited 2004 Apr 20]. Available from; <http:// www.gla.ac.uk>

19. Schlecht NF. Effect of smoking cessation and tobacco type on the risk of causes of the upper aerodigestive tract in Brazil. Epidemiology. 1999;10(4):412-18.

20. Ferlay J, Bray F, Pisani P, Parkin DM. GLOBOCAN 2000: Cancer Incidence, Mortality and Prevalence Worldwide, Version 1.0. IARC Cancer Base no. 5. Lyon: IARC Press; 2001.

21. Andre K, Schraub S, Mercier M, Bontemps P. Role of alcohol and tobacco in the aethiology of head and neck cancer: a case-control study in the Doubs Region of France. Eur J Cancer B Oral Oncol. 1995;31B(5):301-309.

22. Amar A, Franzi AS, Rapoport A, Bisordi C, Lehn CN. Qualidade de vida e prognóstico nos carcinomas epidermóides de cabeça e pescoço. Rev Bras Otorrinolaringol. 2002;68(3):400-403.

23. Neville BW, Day TA. Oral cancer and precancerous lesions. CA Cancer J Clin. 2002;52(4):195-215.

24 . Sciubba JJ. Oral cancer and its early detection: historytaking and the diagnostic phase management. JADA. 2001;132:12S-18S.

25. Costa ALL, Pereira JC, Nunes AAF, Arruda MLS. Correlação entre a classificação TNM, gradação histológica e localização anatômica em carcinoma epidermóide oral. Pesq Odontol Bras. 2002;16(3):216-20.

26. UICC. [homepage on the internet]. TNM Cancer Staging. UICC TNM Prognostic Factors Committees Geneva: International Union Against Cancer; c2004 [cited 2004 Set 12]. Available from: <http://www.uicc.org>

27. Instituto Nacional de Câncer (INCA/MS). [homepage na internet]. Estimativa da incidência e mortalidade por câncer no Brasil. [acesso em set 2006]. Disponível em: <http:// www.inca.gov.br>

28. Robertson AG, Robertson C, Soutar DS, Burns H, Hole D, Mccarron P. Treatment of oral cancer: the need for defined protocols and specialist centres. Variations in the treatment of oral cancer. Clin Oncol. 2001;13:409-15.

29. Adelstein DJ, Li Y, Adams GL, Wagner H Jr, Kish JA, Ensley JF, et al. An Intergroup Phase III Comparison of standard radiation therapy and two schedules of concurrent chemoradiotherapy in patients with unresectable squamous cell head and neck cancer. J Clin Oncol. 2003;1;21(1):92-98.

30. American Cancer Society [homepage on the internet]. Making treatment decisions [cited 2004 Set 5]. Available from: <http://www.cancer.org>

31. Joseph E, Baibak L. Head and neck cancer: squamous cell carcinoma. eMedicine [serial on the internet]. [cited 2006 Apr 26]. Available from: <http://www.emedicine.com/ 
plastic/topic376.htm>

32. Das BR, Nagpal JK. Understanding the biology of oral cancer. Med Sci Monit. 2002;8(11):RA258-67.

33. Vikram B. Adjuvant therapy in the head and neck cancer.
CA Cancer J Clin. 1998;48(4):199-209.

34. Salvajoli JV. Câncer de cabeça e pescoço. In: Salvajoli JV, Souhami L, Faria SL. Radioterapia em oncologia. Rio de Janeiro: MEDSI; 1999:335-68.

\section{Abstract}

Oral squamous cell carcinoma (OSCC) is a malignant neoplasm originating in the oral epithelium and accounting for some $95 \%$ of malignant oral lesions. In Brazil, cancer of the mouth shows the 5th highest cancer incidence in men and the 7th highest in women. This paper reviews the variables gender, age, occupation, schooling, smoking, alcohol consumption, and exposure to sunlight, clinical staging (TNM system), and proposed treatment. Seventyfive articles were included. Lesions were observed most frequently in the tongue, followed by the floor of the mouth. Most cases occurred in white individuals in their fifties to seventies. At the time of diagnosis, most patients had advanced-stage lesions (III or IV). Factors most associated with oral cancer were smoking, alcohol consumption, and exposure to sunlight. These factors can also complicate the natural course and prognosis. Delayed diagnosis was frequent and complicated the initially proposed treatment.

Key words: Oral squamous cell carcinoma; Epidemiology; Patient profile; Clinical staging; Treatment. 